\title{
Riemann Surfaces and Partial Wave Models
}

\author{
R. B. JONES \\ Department of Physics, Queen Mary College, University of London
}

Received July 23, 1969; in revised form January 10, 1970

\begin{abstract}
Within the context of simple partial wave models for elastic scattering the problem of uniformizing the partial wave amplitude and classifying its Riemann surface is studied. Starting with the analytic continuation of the amplitude an analysis of the Riemann surface is made through its group of covering transformations relative to a simpler base surface. A model based on the Yukawa potential is studied in this manner and the Riemann surface of interest is found to be the universal covering surface of the thrice punctured sphere. The uniformization of the amplitude can be done explicitly in this case by use of the elliptic modular function. In terms of the uniformizing variable, the original discontinuity relations for the amplitude then reduce to functional equations involving elements of the modular group.
\end{abstract}

\section{Introduction}

In high energy physics single variable analyticity is of particular usefulness in the study of partial wave amplitudes for four-point functions where the energy is the only complex variable of interest. Through partial wave dispersion relations and the $N / D$ technique, single variable analyticity gives a method of calculating simple partial wave models which can aid the qualitative understanding of many processes. The simplest such models use two-body elastic unitarity together with some assumed left hand cut from which information one must construct a physically sensible amplitude.

Because the partial wave amplitudes are multivalued analytic functions, the analyticity is most succinctly expressed through the structure of the underlying abstract Riemann surface on which the amplitude function is defined. Indeed, in the case of a partial wave model, we may separate the problem of constructing the amplitude from that of studying its Riemann surface. In this paper we will study a particular partial wave model based on the Yukawa potential showing how the classical results of the theory of Riemann surfaces enable one to perform a detailed analysis of the particular surface underlying the model. In this model we will find that the Riemann surface of the amplitude is the universal covering surface of a sphere with three points removed, and hence that the amplitude is uniformized by means of the elliptic modular function. 
We will require only those classical results of Riemann surface theory embodied in the uniformization theorem and in the notion of covering surfaces of a Riemann surface. These results are expounded in detail in several excellent books where full explanations may be found [1-4]. Much of our notation is borrowed from the work by Springer [2].

\section{Analytic Continuation of the Amplitude and the Realization of its Riemann Surface}

Let us consider elastic two-body scattering. A partial wave amplitude for this process is a multivalued analytic function $g(E)$ defined in a cut energy plane (physical sheet) where it has left and right hand cuts on the real energy axis as in Fig. 1. It is further assumed to be real analytic, $g(\bar{E})=\overline{g(E)}$, and meromorphic in the physical sheet.

A partial wave model results if we specify the discontinuity of $g(E)$ across each of its two cuts and then construct an admissible amplitude from this information. The right hand unitary cut occurs at energies where physical scattering takes place. Assuming elastic unitarity or using some inelasticity factor we can write the right hand discontinuity as

$$
\operatorname{Disc} g(E)=g\left(E_{+}\right)-g\left(E_{-}\right)=\sigma(E)|g(E)|^{2} .
$$

The left hand force cut has discontinuity

$$
\operatorname{Disc} g(E)=g\left(E_{+}\right)-g\left(E_{-}\right)=\varrho(E) .
$$

The basic specification of a model then consists of giving the functions $\sigma(E)$ and $\varrho(E)$.

Putting aside the problem of constructing $g(E)$, let us ask on what Riemann surface it is to be defined. If we were given such a function $g(E)$ satisfying the conditions above, the procedure would be clear. Namely, starting from some function element (a convergent power series for $g(E)$ about some point $E$ ) in the physical sheet, we would analytically continue it through both cuts along all possible continuous curves in the E-plane [2]. In this way we would obtain a collection of analytically continued function elements and the ordered pairs $(E, g(E))$,

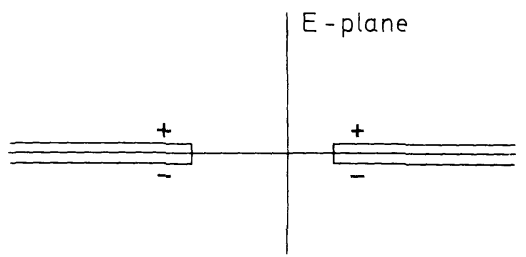

Fig 1 
where $g(E)$ is some continued function element, would provide a standard realization of the Riemann surface desired [2].

The first question then is how to analytically continue a given $g(E)$ through its cuts. The answer is well-known [5] and may be stated in the following manner, where we denote the function elements of $g(E)$ in the physical sheet by $g^{p}(E)$. Assuming $g^{p}(E)$ given in the physical sheet, it can be analytically continued through a point $E_{0}$ on the left hand cut if and only if, inside some small circle about $E_{0}$, the discontinuity function $\varrho(E)$ itself is analytic. The desired continuation, inside that assumed circle, is

$$
g(E)=g^{p}(E) \pm \varrho(E)
$$

where $g^{p}(E)$ is a function element of $g(E)$ at point $E$ in the physical sheet, $g(E)$ is the function element at point $E$ on a sheet reached by going through the left hand cut at $E_{0}$ either from above (+) or below (-), and $\varrho(E)$ is the function element arising from the given left hand discontinuity function at the point $E_{0}$. We may continue through the right hand cut by applying the above result to the real analytic function $\frac{1}{g(E)}$ for which

$$
\operatorname{Disc} \frac{1}{g(E)}=-\sigma(E) \text {. }
$$

on the right hand cut. This result is stated here only for continuation through an infinitesimal neighborhood of a point $E_{0}$ lying on one of the two cuts. To view the Riemann surface of $g(E)$ globally we must do more than this, namely we must continue the given function elements $g^{p}(E)$ over all continuous curves in the $E$-plane. In general, $g(E)$ will possess a countable isolated set of singular function elements where it may have poles or algebraic branch points [2]. However, in principle we can always avoid these by small detours and thus carry out the continuation entirely by means of the regular function elements. Before stating the result of this global continuation let us first rewrite the result in Eq. (3) in a different manner.

For a complex variable $x$, a linear fractional transformation $T x$ is defined by

$$
T x=\frac{a x+b}{c x+d}
$$

where $a, b, c, d$ are complex numbers normalized by the condition $a d-b c=1$. The transformation $T$ may be represented by the unimodular matrix $\left(\begin{array}{ll}a & b \\ c & d\end{array}\right)$ where the same transformation $T$ is specified 
also by the matrix $\left(\begin{array}{cc}-a & -b \\ -c & -d\end{array}\right)$. The composition of two such transformations then corresponds simply to multiplication of the corresponding matrices. Now the result of the analytic continuation (3) may be simply expressed as such a linear fractional transformation:

$$
\begin{aligned}
g(E) & =g^{p}(E) \pm \varrho(E)=L_{ \pm}(E) g^{p}(E) \\
L_{ \pm}(E) & =\left(\begin{array}{cc}
1 & \pm \varrho(E) \\
0 & 1
\end{array}\right) \\
L_{-}(E) & =\left(L_{+}(E)\right)^{-1}
\end{aligned}
$$

Here $L_{ \pm}(E)$ denotes a two-by-two unimodular matrix whose entries are function elements at the point $E$ rather than just complex numbers. Eq. (5) expresses the continuation through the cut as a linear fractional transformation of the physical sheet function element $g^{p}(E)$. Combining (3) and (4), the continuation through the right hand cut is expressed as

$$
\begin{aligned}
g(E) & =\frac{g^{p}(E)}{\mp \sigma(E) g^{p}(E)+1}=R_{ \pm}(E) g^{p}(E), \\
R_{ \pm}(E) & =\left(\begin{array}{ll}
1 & 0 \\
\mp \sigma(E) & 1
\end{array}\right), \\
R_{-}(E) & =\left(R_{+}(E)\right)^{-1},
\end{aligned}
$$

where again $R_{ \pm}(E)$ is a unimodular matrix of function elements. The \pm subscripts on $L$ and $R$ refer to continuing through the respective cuts from above $(+)$ or below $(-)$.

To continue globally we start at some point $E$ in the physical sheet with a given function element $g^{p}(E)$ and then continue it along some continuous curve $c$. Each time the path $c$ crosses a cut $g^{p}(E)$ undergoes a linear fractional transformation as in Eq. (5) or (6). After crossing the cuts several times the continued function element $g(E)$ is expressed, by a succession of transformations, in terms of the physical sheet function element $g^{p}(E)$ and in terms of certain matrices of analytically continued function elements of $\varrho(E)$ and $\sigma(E)$. At the end of the continuation along $c$ we reach a function element

$$
g(E)=T_{1}(E) T_{2}(E) \ldots T_{n}(E) g^{p}(E)
$$

where each $T_{j}(E)$ is one of the transformations $L_{ \pm}(E), R_{ \pm}(E)$. In this expression the order in which the $T_{j}(E)$ occur expresses the order in which the cuts have been crossed on $n$ occassions. Further, $T_{j}(E)$ is a matrix of function elements each of which has itself been analytically continued along the curve $c$ from that point on $\mathfrak{c}$ at which $T_{j}(E)$ arose 
due to crossing a cut. Finally, the function elements $\varrho(E)$ or $\sigma(E)$ appearing in $T_{j}(E)$ have an additional dependence on the point $E^{\prime}$ where the cut was crossed since we can specify $\varrho$ or $\sigma$ to be quite distinct analytic functions on different open connected subsets of the cut.

Ultimately we realize the entire Riemann surface of $g(E)$ by the collection of ordered pairs

$$
(E, g(E))=\left(E, T_{1}(E) T_{2}(E) \ldots T_{n}(E) g^{p}(E)\right)
$$

plus the singular function elements discovered during the analytic continuation. It is evident from (8) that the function elements in the physical sheet $g^{p}(E)$, which we assumed known initially, are dummy function elements in a sense. Namely, by correlating the ordered pairs $\left(E, T_{1}(E) \ldots T_{n}(E)\right)$ together with the curves $\mathfrak{c}$ in the $E$-plane underlying each sequence $T_{1} \ldots T_{n}$, one could directly construct the Riemann surface without referring to $g^{p}(E)$. Thus the structure of the Riemann surface of $g(E)$ may be determined directly from the assumed discontinuity functions in the model, $\varrho(E)$ and $\sigma(E)$, without first constructing the function $g(E)$.

\section{Covering Group Analysis of a Potential Scattering Model}

The realization of the Riemann surface of a model partial wave amplitude through the ordered pairs in (8) is in general not of immediate usefulness because of the complexity which may be involved in the continuation of the matrices $T_{j}(E)$ when the functions $\varrho(E)$ and $\sigma(E)$ have branch points themselves. However, if the model is simple enough, these complexities drop away and we can interpret the realization in terms of covering surfaces. The associated covering groups then allow us to completely characterize the topological and conformal structure of the original Riemann surface.

Let us now illustrate this procedure by analyzing a simple model based on potential scattering. Specifically we ask for an amplitude $g(E)$ with right hand discontinuity due to elastic unitarity,

$$
\sigma(E)=2 i \sqrt{E}, \quad 0 \leqq E \leqq+\infty
$$

with the positive branch of $\sqrt{E}$ chosen. For the left hand discontinuity let us take that arising from the Born approximation for the Yukawa potential $V(r)=g^{2} \frac{e^{-m r}}{r}$ :

$$
\varrho(E)=-i \pi \frac{g^{2}}{E} P_{l}\left(1+\frac{m^{2}}{2 E}\right), \quad-\infty \leqq E \leqq-\frac{m^{2}}{4},
$$


where $P_{l}$ is a Legendre polynomial. Since $\varrho(E)$ is a rational function of $E$ with no branch points, there is no difficulty in continuing $\varrho(E)$ along any curve $\mathfrak{c}$ while continuing the amplitude $g(E)$. However, $\sigma(E)$ has two branch points and must itself be appropriately continued while continuing $g(E)$.

The general function element reached along a curve in the $E$-plane is

$$
g(E)=T_{1}(E) T_{2}(E) \ldots T_{n}(E) g^{p}(E)
$$

where each $T_{j}(E)$ is a linear fractional transformation which is either

$$
L(E)=\left(\begin{array}{cc}
1 & \varrho(E) \\
0 & 1
\end{array}\right) \text { or } \quad R(E)=\left(\begin{array}{cc}
1 & 0 \\
-\sigma(E) & 1
\end{array}\right)=\left(\begin{array}{cc}
1 & 0 \\
-2 i \sqrt{E} & 1
\end{array}\right),
$$

or their inverses $L^{-1}, R^{-1}$. The transformations $L$ and $R$ do not commute. Since $\varrho(E)$ has no branch points, the matrix representing $L(E)$ always has the same form. However the branch points of $\sigma(E)=2 i \sqrt{E}$ cause the matrix for $R(E)$ to depend on the path of continuation. If $R(E)$, or $R^{-1}(E)$ occurs as $T_{j}(E)$ in the sequence $T_{1}(E) \ldots T_{n}(E)$, then the function element of $\sigma(E)$ which appears in $T_{j}(E)$ must be that appropriate function element which has been analytically continued along that part of the curve $c$ corresponding to the appearance of the transforms $T_{j+1}(E) \ldots T_{n}(E)$. We know that $R(E)$ must have one of the two forms

$$
R(E)=\left(\begin{array}{cc}
1 & 0 \\
\mp 2 i \sqrt{|E|} e^{i \frac{0}{2}} & 1
\end{array}\right), \quad \theta=\arg E ，
$$

depending on the branch of $\sqrt{E}$. Thus if the transformation $R(E)$ occurs twice in succession, say as $T_{j}(E) T_{j+1}(E)$ in the sequence $T_{1}(E) \ldots T_{n}(E)$, we will have

$$
T_{j}(E) T_{j+1}(E)=R(E) R(E)=\left(\begin{array}{cc}
1 & 0 \\
\pm 2 i \sqrt{|E|} e^{i \frac{0}{2}} & 1
\end{array}\right)\left(\begin{array}{cc}
1 & 0 \\
\mp 2 i \sqrt{|E| e^{\frac{\theta}{2}}} & 1
\end{array}\right)=\left(\begin{array}{ll}
1 & 0 \\
0 & 1
\end{array}\right),
$$

because $T_{j+1}(E)$ represents an additional passage through the unitarity cut (and an additional circuit of $E=0$ by c) relative to $T_{j}(E)$, producing the sign change of $\sqrt{E}$ in $T_{j}(E)$. So $R(E)$ satisfies a relation

$$
R(E) R(E)=R^{-1}(E) R^{-1}(E)=1
$$

which expresses the familiar fact that $E=0$ is a branch point of order one for the amplitude $g(E)$. Since $L(E)$ has no possibility of such sign changes, no finite number of circuits of $\mathfrak{c}$ around the left hand branch point at $E=-\frac{m^{2}}{4}$ can ever return us to the starting function element. Hence the branch points at $E=-\frac{m^{2}}{4}, \infty$, are logarithmic of infinite order. 
Because the threshold branchpoint at $E=0$ is algebraic of order one, the Riemann surface $\mathscr{R}$ of $g(E)$ is a branched unlimited covering surface [2] of the $E$-sphere with the logarithmic branch points $-\frac{m^{2}}{4}$ and $\infty$ removed. Since $\mathscr{R}$ is branched and not smooth [2] relative to the $E$-sphere, it is simplest to analyze $\mathscr{R}$ in two steps, first considering only the right hand unitarity cut and then dealing separately with the left hand cut. If we ignore the left hand cut by setting $\varrho(E)=0$, then the resulting function $g_{1}(E)$ is only two sheeted, with the points $\left(E, g_{1}^{p}(E)\right)$, and $\left(E, R(E) g_{1}^{p}(E)\right)$ corresponding to the two different sheets of its Riemann surface $\mathscr{R}_{1}$. Now $\mathscr{R}_{1}$ has no logarithmic branchpoints and so is compact. By a standard formula [2] we see that $\mathscr{R}_{1}$ is of genus zero, that is, $\mathscr{R}_{1}$ is conformally equivalent to a sphere $\mathscr{S}$. If we now include the left hand cut with its logarithmic branchpoints, we may regard the Riemann surface $\mathscr{R}$ of $g(E)$ to be a smooth unlimited covering surface of $\mathscr{R}_{1}$, despite the branching of $\mathscr{R}$ relative to the $E$-sphere. In fact, given a point $\left(E, T_{1}(E) \ldots T_{n}(E) g^{p}(E)\right)$ on $\mathscr{R}$, then if an even number of factors $T_{j}(E)$ are either $R(E)$ or $R^{-1}(E)$, define the point to lie over the base point $\left(E, g_{1}^{p}(E)\right)$ on $\mathscr{R}_{1}$, while for an odd number of $T_{j}(E)$ chosen from $R(E)$ or $R^{-1}(E)$, let the point lie over the base point $\left(E, R(E) g_{1}^{p}(E)\right)$ on $\mathscr{R}_{1}$. Relative to the base surface $\mathscr{R}_{1}, \mathscr{R}$ now has no algebraic branchpoints, rather it has branch points of infinite order at the three points $\left(-\frac{m^{2}}{4}, g_{1}^{p}\left(-\frac{m^{2}}{4}\right)\right)$, $\left(-\frac{m^{2}}{4}, R\left(-\frac{m^{2}}{4}\right) g_{1}^{p}\left(-\frac{m^{2}}{4}\right)\right)$, and $\left(\infty, g_{1}^{p}(\infty)\right)$ of $\mathscr{R}_{1}$. Excluding these three points and remembering the conformal equivalence above, we see that $\mathscr{R}$ is a smooth unlimited covering surface of some thrice punctured sphere $\mathscr{S}_{1}$.

Since $\mathscr{R}$ is a regular covering surface of $\mathscr{R}_{1}$, we may now ask for the cover group $\mathfrak{H}$ of $\mathscr{R}$ relative to the base surface $\mathscr{R}_{1}[2,3]$. In accord with our definition above of $\mathscr{R}$ as a covering surface of $\mathscr{R}_{1}$, a cover transformation $\mathfrak{h}_{1}$ of $\mathfrak{H}$ may be specified by

$$
(E, g(E)) \stackrel{\mathfrak{h}_{1}}{\longrightarrow}\left(E, T_{1}(E) T_{2}(E) \ldots T_{n}(E) g(E)\right)
$$

where $T_{1}(E) \ldots T_{n}(E)$ is any finite sequence chosen from $L(E), R(E)$, $L^{-1}(E)$, or $R^{-1}(E)$ such that only an even number of the $T_{j}(E)$ are selected from the pair $R(E), R^{-1}(E)$. Defining composition of two such transformations in an obvious way, we clearly get a group which serves to permute all the points of $\mathscr{R}$ lying above any one given base point in $\mathscr{R}_{1}$. Using relation (10) any such sequence $T_{1}(E) \ldots T_{n}(E)$ can be rewritten as a finite product of the two elements $L(E)$ and $R(E) L(E) R^{-1}(E)$. Conversely, any finite product of these two will give an element of the 


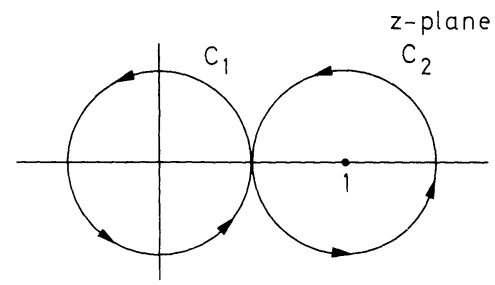

Fig. 2

covering group. Thus the covering group $\mathfrak{H}$ of $\mathscr{R}$ relative to $\mathscr{R} 1$ is a nonabelian group generated by the two non-commuting elements $L(E)$ and $R(E) L(E) R^{-1}(E)$.

Next let us examine the base surface $\mathscr{R}_{1}$ which is conformally equivalent to a sphere $\mathscr{S}$. The mapping which takes $\mathscr{R}_{1}$ onto $\mathscr{S}$ is the obvious mapping from potential scattering, $k=\sqrt{E}$. $\mathscr{R}$ then is a smooth unlimited covering surface of the $k$-sphere with the three points $k=i \frac{m}{2},-i \frac{m}{2}, \infty$ removed. We may normalize the $k$-sphere by mapping $-i \frac{m}{2}, i \frac{m}{2}, \infty$ respectively onto $0,1, \infty$ in the $z$-sphere

$$
z=-\frac{i}{m} k+\frac{1}{2}, \quad k=i m\left(z-\frac{1}{2}\right) .
$$

Finally, let us denote by $\mathscr{S}_{1}$ this thrice punctured $z$-sphere with the points $z=0,1, \infty$ omitted. By inspection we see that the fundamental group $\mathfrak{F}_{1}$ of $\mathscr{S}_{1}$ may be generated by the two curves $\mathfrak{c}_{1}, \mathfrak{c}_{2}$ shown in Fig. 2. $\mathfrak{c}_{1}$ and $\mathfrak{c}_{2}$ start from the same point and enclose respectively the excluded points $z=0,1$ in a counter-clockwise sense. One sees that $\mathfrak{F}_{1}$ is a non-abelian group generated by two non-commuting elements $\mathfrak{c}_{1}$ and $\mathfrak{c}_{2}$. Thus the identification $L(E) \leftrightarrow \mathfrak{c}_{2}, R(E) L(E) R^{-1}(E) \leftrightarrow \mathfrak{c}_{1}$ gives an explicit isomorphism between $\mathfrak{H}$, the cover group of $\mathscr{R}$, and $\mathfrak{F}_{1}$, the fundamental group of the base surface $\mathscr{S}_{1}$. The cover group $\mathfrak{S}$ is transitive [2], that is given any two points on $\mathscr{R}$ above the same base point on $\mathscr{S}_{1}$, there is an obvious element $\mathfrak{h}_{1}$ of $\mathfrak{H}$ which carries one point onto the other. Therefore, by standard theorems on the covering groups associated with regular covering surfaces $[2,3]$, since $\mathfrak{H}$ is isomorphic to $\mathfrak{F}_{1}$, we may conclude that $\mathscr{R}$ is the universal covering surface of $\mathscr{S}_{1}$.

To obtain $\mathscr{R}$ and $\mathscr{T}_{1}$ in a canonical representation we can now use the uniformization theorem $[1,2]$ which tells us that we may map the universal covering surface of the thrice punctured $z$-sphere one-to-one and conformally onto some open upper half $\omega$-plane. This new variable $\omega$ will now serve as a global coordinate on both $\mathscr{R}$ and the base surface $\mathscr{S}_{1}$. Because many points of $\mathscr{R}$ lie above each point of $\mathscr{S}_{1}$, this mapping 
will be a one-to-many mapping of the punctured $z$-sphere onto the upper half $\omega$-plane, with a countable sequence of points $\omega_{j}$ corresponding to each point $z$. This mapping is classical; in terms of $\omega$ and $z$, it is given by the elliptic modular function $[2,6,7,9]$.

$$
\omega=\lambda^{-1}(z)=i \frac{{ }_{2} F_{1}\left(\frac{1}{2}, \frac{1}{2} ; 1 ; 1-z\right)}{{ }_{2} F_{1}\left(\frac{1}{2}, \frac{1}{2} ; 1 ; z\right)} .
$$

Here ${ }_{2} F_{1}$ denotes the hypergeometric function. The elliptic modular function itself, $\lambda(\omega)$, can be expressed in terms of theta functions $[6,8]$

$$
z=\lambda(\omega)=\left[\frac{\Theta_{2}(\omega)}{\Theta_{3}(\omega)}\right]^{4} .
$$

The function $\lambda(\omega)$ has the real $\omega$-axis as a natural boundary and takes all complex values except 0,1 , and $\infty$ in the open upper half $\omega$-plane.

Since $\mathscr{R}$ was not only a covering surface of $\mathscr{S}_{1}$, but also the Riemann surface of our amplitude $g(E)$, the variable $\omega$ uniformizes $g(E)$. That is, in terms of $\omega, g(E)=g(E(\omega))=g(\omega), g(\omega)$ is meromorphic in the upper half $\omega$-plane with no branch points remaining. Before sketching the mapping from $E$ to $\omega$, let us briefly quote a few properties of $\lambda(\omega)$. Since many values $\omega_{j}$ correspond to the same $z$ value, we expect $\lambda(\omega)$ to be automorphic with respect to a discontinuous group of linear fractional transformations preserving the upper half $\omega$-plane [6]. This group is $\Gamma(2)$, a principal congruence subgroup of the modular group $\Gamma(1)$ [6]. $\Gamma(2)$ is simply described as the group of linear fractional transformations of the variable $\omega$ generated by the two basic transformations

$$
S=\left(\begin{array}{ll}
1 & 2 \\
0 & 1
\end{array}\right), \quad V=\left(\begin{array}{rr}
1 & 0 \\
-2 & 1
\end{array}\right)
$$

One has $z=\lambda(\omega)=\lambda(S \omega)=\lambda(V \omega)$ and thus $\lambda(\omega)$ is indeed automorphic on $\Gamma(2)$.

A canonical representation of $\mathscr{S}_{1}$ is given by the quotient space $\frac{H}{\Gamma(2)}$ of the open upper half $\omega$-plane $H$ with respect to the group $\Gamma(2)$. This quotient space is easily visualized as in Fig. 3. The unshaded region there including sides 1 and 2 , but excluding sides 3 and 4 , is a fundamental region for $\Gamma(2)$; that is, it contains no two points equivalent under $\Gamma(2)$ while any point of $H$ outside this region is equivalent to one point inside [6]. The transformation $S$ takes side 4 onto side 1, while $V$ carries side 2 onto side 3 . Under the whole group $\Gamma(2)$ the fundamental region is translated in such a way as to cover completely the upper half $\omega$-plane [6]. The quotient space $\frac{H}{\Gamma(2)}$ is simply the fundamental region with 


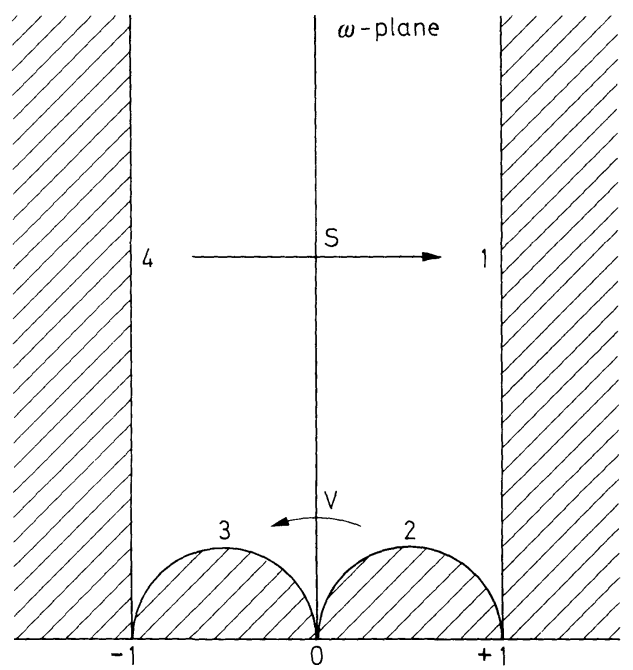

Fig. 3

sides 1 and 4 and sides 2 and 3 identified. This clearly produces a thrice punctured sphere $\mathscr{S}_{1}$, the points left out corresponding to $\omega=0,1, i \infty$. One may show that $\lambda(\omega)$ takes each complex value exactly once in the fundamental region except for $0,1, \infty$ which are the limiting values of $\lambda(\omega)$ if we take the following limits from within the fundamental region, $\omega \rightarrow i \infty, \omega \rightarrow 0, \omega \rightarrow \pm 1[6,8]$. The group $\Gamma(2)$ is isomorphic to the cover group $\mathfrak{H}$ and also to $\mathfrak{F}_{1}$, the fundamental group of $\mathscr{S}_{1}$, by the identification $V \leftrightarrow L(E), S \leftrightarrow R(E) L(E) R^{-1}(E)$.

The uniformizing map which takes us from $E$ to $\omega$ is given by the equations

$$
\begin{aligned}
& z=\lambda(\omega), \\
& k=i m\left(z-\frac{1}{2}\right)=i m\left(\lambda(\omega)-\frac{1}{2}\right), \\
& E=k^{2}=-m^{2}\left(\lambda(\omega)-\frac{1}{2}\right)^{2} .
\end{aligned}
$$

The effect of this mapping is illustrated in Figs. 4 and 5. Having reduced the Riemann surface $\mathscr{R}$ of $g(E)$ to its canonical form as an upper half plane, we can now rewrite the discontinuity relations for $g(E)$. Since the uniformizing mapping has separated the two sides of the cuts in both the $E$ and $k$ planes, the left hand discontinuity relation becomes a functional equation relating the values of $g(\omega)$ on different sides of the fundamental region,

$$
g(\omega)-g(S \omega)=g(\omega)-g(V \omega)=\frac{i \pi g^{2}}{E(\omega)} P_{l}\left(1+\frac{m^{2}}{2 E(\omega)}\right) .
$$



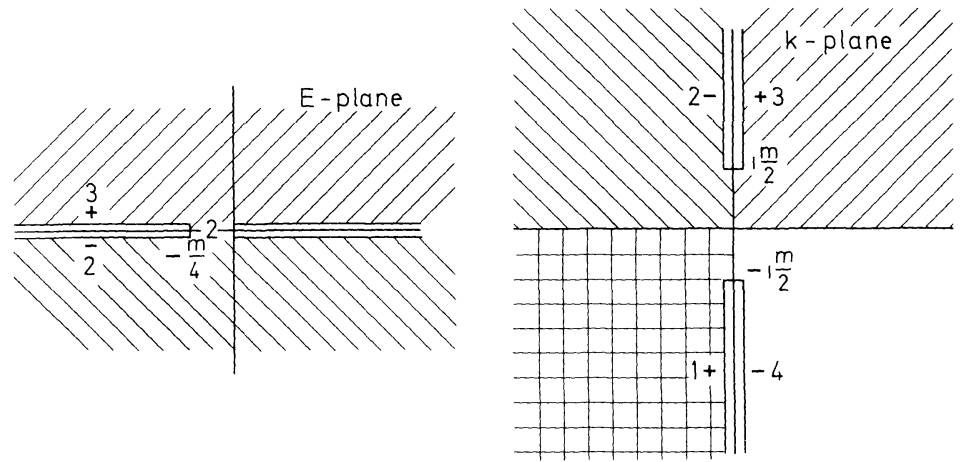

Fig. 4

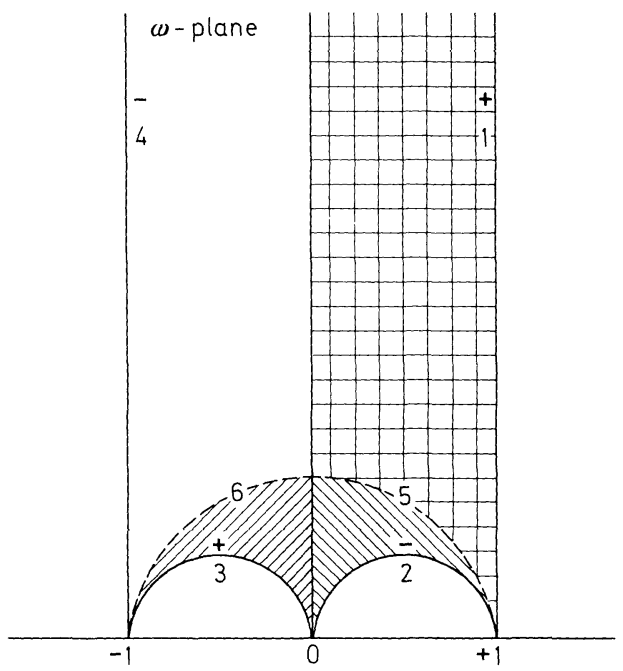

Fig. 5

Since $g(\omega)$ is meromorphic in $\omega,(15)$ is valid for all $\omega$ in the upper half plane, not just for $\omega$ on the sides of the fundamental region. To express the right hand unitarity discontinuity we require an extra linear fractional transformation of the upper half $\omega$-plane,

$$
W=\left(\begin{array}{rr}
0 & -1 \\
1 & 0
\end{array}\right)
$$

The transformation $W$, a generator of the group $\Gamma(1)$, corresponds to the transformation of function elements $R(E)$ which arose on analytically continuing through the unitarity cut. Indeed, $V$ and $S=W V W^{-1}$ correspond directly to $L(E)$ and $R(E) L(E) R^{-1}(E)$. As a transformation 
on the upper half $\omega$-plane $W$ interchanges the two pieces of the half plane separated by the dotted semicircle in Fig. 5, mapping the semicircle onto itself, $5 \leftrightarrow 6$. Using $W$ the unitarity discontinuity equation becomes

$$
\frac{1}{g(\omega)}-\frac{1}{g(W \omega)}=-2 i \sigma(E(\omega))=m(2 \lambda(\omega)-1) .
$$

The three equations of (15) and (17) are non-linear functional equations for $g(\omega)$. A Jost function ansatz linearizes the equations. Setting

$$
g(E)=\frac{S(E)-1}{2 i \sqrt{E}} \text { and } S(E)=S(k)=\frac{f(k)}{f(-k)},
$$

where $S(E)$ is the $S$-matrix element and $f(k)$ is the Jost function, we identically satisfy unitarity (17), while the two Eqs. (15) become

and

$$
f(\omega)-f(V \omega)=\frac{2 \pi g^{2}}{k(\omega)} P_{l}\left(1+\frac{m^{2}}{2 E(\omega)}\right) f(W \omega),
$$

$$
f(\omega)-f(S \omega)=0
$$

\section{Conclusion}

We have shown in this paper that in the simplest partial wave models the assumed discontinuity relations for the amplitude already contain the structure of the underlying Riemann surface. In particular, we have demonstrated in a simple model how the analytic continuation off the physical sheet leads to a classification of the Riemann surface based on certain covering groups. In the model considered, this classification also enabled us to explicitly uniformize the amplitude $g(E)$ through a classical mapping.

In principle, the methods used could be applied to much more complicated models. However, the covering group description and the uniformizing procedure become difficult to write out explicitly for even moderately more complexity in the discontinuity relations. One exception is the simplest relativistic generalization of the model discussed here, namely equal mass elastic scattering with single particle exchange and elastic unitarity. In this case also the Riemann surface and its uniformization can be analyzed relatively simply and explicitly. This procedure will be described elsewhere.

In models where the uniformization can be done explicitly, the more practical problem arises of whether the model can be most usefully approximated in the $E$ variable or in the uniformizing $\omega$ variable. In some contexts it is helpful to employ a uniformizing variable for the 
unitarity cut alone, such as $k=\sqrt{\mathrm{E}}$ in potential scattering, or appropriate generalizations in relativistic situations [10]. However, by uniformizing the amplitude with respect to both cuts, one has the possibility of approximating the model amplitude in such a way as to preserve completely the topological and conformal nature of the underlying Riemann surface, a procedure in marked contrast to the usual pole approximation to the left hand cut in terms of the $E$ variable. We hope to study this question further for a soluble model similar to that studied above.

\title{
References
}

1. Weyl, H.: The concept of a Riemann surface, 3rd Ed.Reading, Mass.: AddisonWesley 1955.

2. Springer, G.: Introduction to Riemann surfaces. Reading, Mass.: Addison-Wesley 1957.

3. Ahlfors, L. V., Sarı, L.: Riemann surfaces. Princeton N. J.: Princeton University Press 1960.

4. Behnke, H., Sommer, F.: Theorie der analytischen Funktionen eines komplexen Veränderlichen, 3. Aufl. Berlin-Heidelberg-New York: Springer 1965.

5. Oehme, R.: Phys. Rev. 121, 1840 (1961).

6. Lehner, J.: Discontinuous groups and automorphic functions. Mathematical Surveys, Number VIII, American Mathematical Society (1964).

7. Higher Transcendental functions, Vol. I, p. 99, Bateman Manuscript Project. New York: McGraw-Hill 1953.

8. Whittaker, E. T., Watson, G. N.: A course of modern analysis, Chapter XXI. Cambridge: Cambridge University Press 1965.

9. Bellman, R.: A brief introduction to theta functions. New York: Holt, Rinehart \& Winston 1961.

10. Wanders, G., Piguet, O.: Nuovo Cimento LVI A, 417 (1968).

\author{
R. B. Jones \\ Department of Physics \\ Queen Mary College \\ University of London \\ Mile End Road \\ London, E. 1, Great Britain
}

